\title{
Delayed Umbilical Cord Clamping is Not Associated with Acute Kidney Injury in Very Low Birth Weight Neonates
}

\author{
Matthew W. Harer, MD ${ }^{1}$ \\ Ryan M. McAdams, MD ${ }^{1}$ \\ Dylan M. Hyatt, $\mathrm{MS}^{3}$ Jennifer R. Charlton, MD ${ }^{3}$ \\ ${ }^{1}$ Department of Pediatrics, University of Wisconsin School of \\ Medicine and Public Health, Madison, Wisconsin \\ ${ }^{2}$ Department of Public Health Sciences, University of Virginia, \\ Charlottesville, Virginia \\ ${ }^{3}$ Department of Pediatrics, University of Virginia, Charlottesville, Virginia \\ Am J Perinatol 2020;37:210-215.
}

\author{
Mark Conaway, $\mathrm{PhD}^{2}$ Brooke D. Vergales, $\mathrm{MD}^{3}$
}

Address for correspondence Matthew W. Harer, MD, 1010 Mound Street, McConnell Hall, 4th Floor, Madison, WI 53715

(e-mail: mwharer@wisc.edu).

\author{
Abstract \\ Keywords \\ - acute kidney injury \\ - delayed cord \\ clamping \\ - umbilical cord \\ milking \\ - very low birth weight \\ - premature \\ - preterm birth \\ - neonate
}

Objective This study aimed to determine if delayed cord clamping (DCC) is associated with a reduction in neonatal acute kidney injury (AKI).

Study Design A retrospective single-center cohort study of 278 very low birth weight (VLBW) neonates was performed to compare the incidence of AKI in the following groups: immediate cord clamping (ICC), DCC, and umbilical cord milking. AKI was diagnosed by the modified neonatal Kidney Diseases and Improving Global Outcomes (KDIGO) definition.

Results The incidence of AKI in the first week was $20.1 \%$ with no difference between groups $(p=0.78)$. After adjustment for potential confounders, the odds of developing AKI, following DCC, compared with ICC was 0.93 (confidence interval [CI]: 0.46-1.86) with no reduction in the stage of AKI between groups.

Conclusion In this study, DCC was not associated with a reduced rate of AKI in VLBW neonates. However, the data suggest that DCC is also not harmful to the kidneys, further supporting the safety of DCC in VLBW neonates.
After birth, preterm newborns undergo a dynamic postnatal transition characterized by significant circulatory and respiratory system alterations. This postnatal transition period may be augmented by delayed umbilical cord clamping (DCC) for 30 to 60 seconds, a placental-to-neonatal transfusion strategy associated with increased newborn blood volumes, arterial oxygen content, cardiac output, and oxygen delivery. ${ }^{1}$ The practice of DCC among preterm births endorsed by the American Academy of Pediatrics, ${ }^{2}$ the American College of Obstetrics and Gynecology, ${ }^{3}$ and International Liaison Committee on Resuscitation ${ }^{4}$ is supported by findings of a recent systematic review and metaanalysis by Fogarty et al which included 18 randomized controlled trials $(2,834$ infants born $<37$ weeks' gestation)

received

May 9, 2019

accepted after revision

August 16, 2019

published online

October 13, 2019 and demonstrated that DCC reduced hospital mortality compared with immediate cord clamping (ICC). ${ }^{5}$ An alternative to DCC is umbilical cord milking (UCM) in which the intact unclamped umbilical cord is stripped three to four times toward the neonate to promote blood transfer before the cord is clamped. ${ }^{1}$ While DCC and UCM appear to be safe in preterm neonates, the impact of these placental transfusion strategies on numerous common preterm neonatal morbidities, including kidney dysfunction, remains unknown. ${ }^{6,7}$

In 2013, the National Institutes of Health recognized the need for a better understanding of acute kidney injury (AKI) in neonates and convened a workshop to discuss controversies surrounding this neonatal morbidity. ${ }^{8}$ The result of this

Copyright @ 2020 by Thieme Medical Publishers, Inc., 333 Seventh Avenue, New York, NY 10001, USA. Tel: +1(212) 584-4662.
DOI https://doi.org/ 10.1055/s-0039-1697671. ISSN 0735-1631. 
workshop was a proposal to utilize the Kidney Diseases and Improving Global Outcomes (KDIGO) modified neonatal AKI criteria. The committee recognized the shortcomings of this definition in neonates including the effect of maternal creatinine on early neonatal creatinine values. Therefore, the definition of AKI requires a rise in creatinine after birth $(0.3 \mathrm{mg} / \mathrm{dL}$ in 48 hours) not an elevated creatinine alone just after birth. Since that time, attention has been focused on the short- and longterm complications of neonatal AKI utilizing the KDIGO's definition of AKI , including several publications from the Neonatal Kidney Collaborative's retrospective study AWAKEN (Assessment of Worldwide Acute Kidney Injury Epidemiology in Neonates). ${ }^{9}$ This multicenter, multinational study demonstrated that neonatal AKI is independently associated with poor short-term outcomes, such as death and length of stay and the risk factors for AKI vary depending on both gestational and postnatal age. ${ }^{10,11}$ Many of the risk factors for neonatal AKI in the first week after birth are related to prerenal causes of AKI, including respiratory distress and mechanical ventilation, hypotension, sepsis, and a hemodynamically significant patent ductus arteriosus. ${ }^{12}$ DCC may affect several of these prerenal AKI causes, since DCC increases circulating blood volume during transitional physiology at birth and decreases rates of neonatal anemia. ${ }^{5,13}$ With no current medications available to treat neonatal AKI, DCC may provide a preventative strategy to reduce the incidence of AKI.

The aim of this study was to determine the relationship of DCC and neonatal AKI in the first week after birth. We hypothesized that DCC would be associated with a lower incidence of AKI compared with ICC.

\section{Materials and Methods}

A retrospective, single-center cohort study of very low birth weight (VLBW) neonates was conducted at the University of Virginia (UVA) neonatal intensive care unit (NICU) from January 1, 2013 to December 31, 2016. During this period, the type of umbilical cord management was determined by the clinician and the decision to use ICC, DCC, or UCM was highly variable between clinicians.

For inclusion, neonates had to be born at UVA and weigh $<1,500 \mathrm{~g}$ at birth. Neonates missing the data to classify their type of umbilical cord care, including those born at other institutions and those with a combination of cord care, were excluded. The primary exposure was DCC, defined as not clamping the umbilical cord for 30 to 90 seconds after delivery, compared with the ICC group, defined as cord clamping that occurred $<15$ seconds after birth. UCM was performed at the discretion of the provider and most frequently in neonates born with poor respiratory effort or tone who were deemed to require immediate resuscitation by the neonatal team. We elected to examine UCM as a separate exposure and defined it as milking a $20-\mathrm{cm}$ segment of umbilical cord three to four times, while the placenta was still attached.

The outcome of interest was AKI defined by the modified KDIGO neonatal AKI staging system which is based on serum creatinine ( $\mathrm{sCr}$ ) and urine output (UOP) values ( - Table 1 ). ${ }^{14}$ AKI was assessed during the first 7 days after birth. Mea-
Table 1 Modified KDIGO neonatal AKI staging

\begin{tabular}{|l|l|l|}
\hline Stage & Serum creatinine & Urine output \\
\hline 0 & $\begin{array}{l}\text { No change in } \mathrm{sCr} \text { or } \\
\text { rise }<0.3 \mathrm{mg} / \mathrm{dL}\end{array}$ & $\begin{array}{l}\text { No changes or decrease } \\
\text { but still }>1 \mathrm{~mL} / \mathrm{kg} / \mathrm{h}\end{array}$ \\
\hline 1 & $\begin{array}{l}\mathrm{sCr} \text { rise } \geq 0.3 \mathrm{mg} / \mathrm{dL} \\
\text { within } 48 \text { hours } \\
\text { or } \mathrm{sCr} \text { rise } \geq 1 \cdot 5-1.9 \times \\
\text { reference } \mathrm{sCr} \\
\text { within } 7 \text { days }\end{array}$ & $>0.5$ and $\leq 1 \mathrm{~mL} / \mathrm{kg} / \mathrm{h}$ \\
\hline 2 & $\begin{array}{l}\mathrm{sCr} \text { rise } \geq 2 \text { to } 2.9 \times \\
\text { reference } \mathrm{sCr}\end{array}$ & $>0.3$ and $\leq 0.5 \mathrm{~mL} / \mathrm{kg} / \mathrm{h}$ \\
\hline 3 & $\begin{array}{l}\mathrm{sCr} \text { rise } \geq 3 \times \text { reference } \\
\mathrm{sCr} \text { or } \mathrm{sCr} \geq 2.5 \mathrm{mg} / \mathrm{dL}^{\mathrm{b}} \\
\text { or receipt of dialysis }\end{array}$ & $\leq 0.3 \mathrm{~mL} / \mathrm{kg} / \mathrm{h}$ or Anuria \\
\hline
\end{tabular}

Abbreviations: AKI, acute kidney injury; KDIGO, kidney diseases and improving global outcomes; $\mathrm{sCr}$, serum creatinine.

Note: KDIGO neonatal AKI definition. Baseline creatinine was the lowest previous value.

a Reference $\mathrm{sCr}$ is the lowest prior $\mathrm{sCr}$ measurement.

${ }^{\mathrm{b}}$ This is lower than the original KDIGO definition as a $\mathrm{SCr}$ of $2.5 \mathrm{mg} / \mathrm{dL}$ in neonates suggests a glomeruluar filtration rate $<10 \mathrm{~mL} / \mathrm{min} / 1.73 \mathrm{~m}^{2}$.

surement of $\mathrm{sCr}$ was performed at the UVA clinical laboratory using the alkaline picrate (Jaffe) method traceable to isotope dilution mass spectrometry. Bedside nurses assessed UOP in 6-hour intervals by weighing diapers.

Perinatal data collected from each neonate included gestational age, sex, delivery type, multiple gestation, Apgar's scores at 1 and 5 minutes, and indomethacin and gentamicin exposure. To assess the degree of illness on admission, Clinical Risk Index for Babies (CRIB)-II scores were collected. Maternal data on preeclampsia, chorioamnionitis, magnesium, and betamethasone exposure were also collected. The presence of intraventricular hemorrhage (IVH; $\geq$ Papile's grade 1 ), necrotizing enterocolitis ( $\geq$ Bell's stage 2 ) and patent ductus arteriosus requiring medication or surgical treatment were recorded. To assess for clinically significant hypotension, exposure to vasopressor medications (dopamine, dobutamine, epinephrine, norepinephrine, and vasopressin) was recorded. To assess blood volume status an initial hematocrit was typically collected within an hour after birth and a follow-up hematocrit within 48 to 72 hours. The number of blood transfusions in the first 28 days was also collected.

All data were gathered from the neonate's electronic medical record and both cord management and laboratory testing were performed at the discretion of the primary team. The $\mathrm{sCr}$ measurements were obtained as part of the standard basic metabolic panel that was obtained daily for the first 7 days after birth.

Statistical analysis for continuous variables was summarized by the median and lower and upper quartiles; the nonparametric Kruskal-Wallis test was used to compare these characteristics across groups. Categorical patient demographic and medical characteristics were tabulated and compared among cord care groups using Chi-squared tests. The Chi-squared test was also used to compare the "early AKI" and "any AKI" outcomes among cord-care groups. Logistic regression was used to identify significant predictors for each definition of AKI. Several 
methods of adjustment were used to account for differing sets of significant predictors for the different definitions. Additionally, for each definition of AKI, stepwise logistic regression was used to select the variables to be included in the model. In addition, logistic regression was used to estimate this odds ratio after adjusting for each individual maternal and neonatal factor. Bayesian model averaging was used to combine these estimates into a single odds ratio estimate. Analyses were performed in SAS version 9.4 (Cary, NC).

\section{Results}

During the study period, 299 VLBW neonates were born at UVA. Of the 299 eligible patients, 21 were excluded due to missing data or the use of combined cord management techniques prior to resuscitation ( - Fig. 1). For the 278 included in the analysis, $45 \%$ received DCC, $42 \%$ had ICC, and $13 \%$ received UCM. The birth weight and gestational age did not differ among the three groups ( - Table 2). The UCM group had a nonstatistically significant higher percentage of extremely preterm infants ( $\leq 27{ }^{6 / 7}$ weeks: $\mathrm{UCM}=59 \%, \mathrm{ICC}=53 \%, \mathrm{DCC}=43 \%$; $p=0.15)$, but statistically significant lower $1-(p<0.001)$ and 5-minute Apgar's scores $(p=0.002)$ and more patients with IVH $(43 \%, p=0.04)$. The ICC group received more days of gentamicin and the UCM group had more exposure to vasopressor medications. There were no statistically significant differences in CRIB-II scores between groups (DCC $=9$ [range, $6-11$ ], ICC $=9$ [range, 6-13], and UCM $=10$ [7-13], $p=0.105$ ).

The initial hematocrit (obtained $<4$ hours after birth) was the highest in the ICC group (ICC $=45.7, \mathrm{DCC}=44.6 ; p=0.02$ ). However, by 48 to 72 hours, the DCC group had the highest hematocrit $(\mathrm{ICC}=42.0, \mathrm{DCC}=43.5 ; p=0.051$ ). In the first 28 days of hospitalization, the DCC group required the fewest number of transfusions $(p=0.01)$. With regards to renal function, the initial $\mathrm{sCr}$ did not differ $(0.7 \mathrm{mg} / \mathrm{dL})$ and the peak $\mathrm{sCr}$ was not different $(0.8-0.9 \mathrm{mg} / \mathrm{dL}, p=0.58)$ among the three groups.

The primary outcome of AKI occurred in 20.1\% $(n=56)$ of neonates. The three cohorts did not differ in AKI incidence $(\mathrm{DCC}=20 \%, \mathrm{ICC}=21.6 \%, \mathrm{UCM}=16.2 \% ; p=0.779$, - Table 3 ). Of the 56 neonates with AKI, only 13 had changes in both $\mathrm{sCr}$

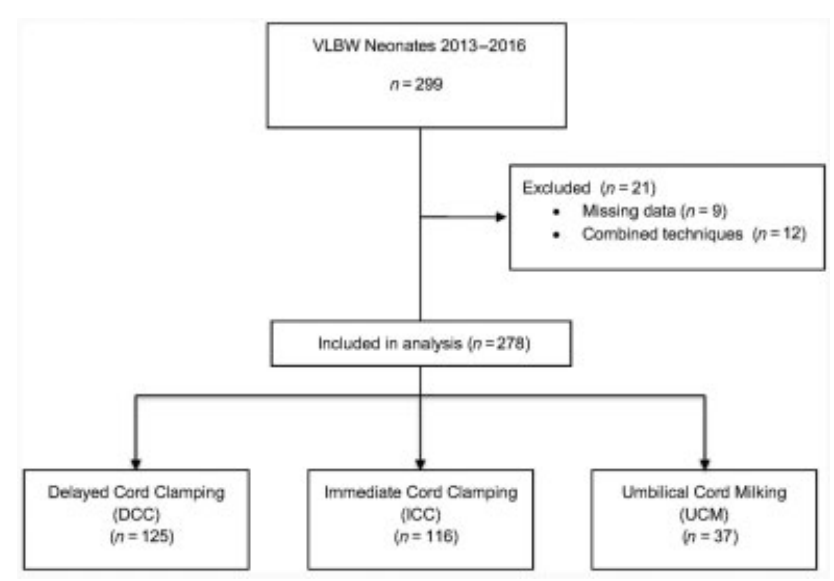

Fig. 1 Study flow chart. VLBW, very low birth weight. and UOP that qualified for AKI. When breaking down the diagnosis of AKI by $\mathrm{sCr}$ or UOP, there were again no differences in three groups. Of the 56 patients with AKI, the majority experienced stage- 1 injury $(n=50)$, while only six patients had stage- 2 injury. There were no statistical differences in severity of injury between the three groups $(p=0.36)$. Additionally, the AKI incidence for the entire hospitalization was not different between the three groups $(\mathrm{DCC}=30.4 \%, \mathrm{ICC}=36.2 \%, \mathrm{UCM}=35.1 \% ; p=0.62)$.

Univariate logistic regression analysis comparing DCC to ICC identified birth weight and the number of blood transfusions within 28 days as significant for every definition of AKI. Subsequent multivariate logistic regression analysis, stepwise logistic regression, and Bayesian's modeling averaging showed no significant difference in the odds of developing AKI when comparing the DCC and ICC groups (- Table 4).

\section{Discussion}

In this retrospective cohort study, we compared the outcome of AKI between VLBW infants who had different cord management styles immediately after birth. Contrary to our hypothesis, the DCC group did not have less AKI in the first week of age when compared with the ICC group. Even after adjusting for significant AKI risk factors, there was no difference between the groups. Although nearly all pediatric and obstetric national and international organizations have begun to recommend DCC, they did so with very little data on how DCC affects kidney function. The current study data provide evidence of safety for DCC from a kidney perspective in VLBW infants who typically have an AKI rate of $29 \%$ during their NICU hospitalization. ${ }^{10}$ With recent evidence from pediatric studies that fluid overload increases the risk of $\mathrm{AKI}^{15}$ and with DCC providing nearly an equivalent of a blood transfusion, this is an important addition to the safety profile of DCC that has not been analyzed in prior studies.

Systematic reviews summarizing the exponential increase in publications and research on DCC compared with ICC in the past 10 years have focused primarily on comparing the risks of mortality and the preterm morbidities of the cerebral, cardiovascular, and hematologic systems. ${ }^{5}$ Few studies have examined the effect on kidney function when comparing ICC to DCC strategies with no studies assessing the risk for AKI. In 1966, Oh and colleagues evaluated a group of female term infants comparing 22 ICC infants to 47 DCC infants. ${ }^{16}$ They found that, in the first 12 hours after birth, the DCC infants had higher UOP and more effective renal blood flow compared with the ICC group. There was a positive correlation between DCC and glomerular filtration rates; however, this did not reach statistical significance. In our study, we similarly did not find any difference in initial sCr's in the three groups, and we also did not have any difference in AKI diagnosis by UOP. It is important to note that the cohort by Oh et al was the study of full term, not preterm, and 50 years separates the studies, making it difficult to make direct comparisons. In the future, new technologies, such as renal near-infrared spectroscopy and functional echocardiography could be used as surrogates for effective renal blood flow in the first 48 hours after birth to compare the effects of DCC, ICC, and UCM on renal blood flow. 
Table 2 Patients' characteristics

\begin{tabular}{|c|c|c|c|c|}
\hline & $\begin{array}{l}\mathrm{DCC} \\
n=125\end{array}$ & $\begin{array}{l}\text { ICC } \\
n=116\end{array}$ & $\begin{array}{l}\text { UCM } \\
n=37\end{array}$ & $p$-Value \\
\hline \multicolumn{5}{|l|}{ Maternal factors } \\
\hline Maternal age (wk) & $28(25-34)$ & $27(23-32)$ & $29(24-35)$ & 0.07 \\
\hline C-section & 87 (70\%) & $82(71 \%)$ & $28(76 \%)$ & 0.77 \\
\hline Multiple birth & $38(30 \%)$ & $33(28 \%)$ & $6(16 \%)$ & 0.23 \\
\hline Chorioamnionitis & $3(2 \%)$ & $3(2 \%)$ & $0(0 \%)$ & 0.62 \\
\hline Preeclampsia & $35(28 \%)$ & $20(17 \%)$ & $9(24 \%)$ & 0.14 \\
\hline Magnesium & 99 (79\%) & $91(78 \%)$ & $27(73 \%)$ & 0.72 \\
\hline Betamethasone doses & & & & 0.07 \\
\hline 0 & $7(6 \%)$ & $10(9 \%)$ & $5(4 \%)$ & \\
\hline 1 & $30(24 \%)$ & $38(33 \%)$ & $6(16 \%)$ & \\
\hline 2 & $88(70 \%)$ & $67(58 \%)$ & $26(70 \%)$ & \\
\hline \multicolumn{5}{|l|}{ Neonatal factors } \\
\hline \multicolumn{4}{|l|}{ Gestational age (wk) } & \multirow[t]{4}{*}{0.15} \\
\hline$\leq 276 / 7$ & $54(43 \%)$ & $61(53 \%)$ & 22 (59\%) & \\
\hline $28-316 / 7$ & $58(46 \%)$ & $38(33 \%)$ & $12(32 \%)$ & \\
\hline$>316 / 7$ & $13(10 \%)$ & $17(15 \%)$ & $3(8 \%)$ & \\
\hline \multicolumn{4}{|l|}{ Birth weight $(\mathrm{g})$} & \multirow[t]{4}{*}{0.31} \\
\hline$<750$ & 24 (19\%) & $30(26 \%)$ & $12(32 \%)$ & \\
\hline $750-1,000$ & $32(26 \%)$ & $26(22 \%)$ & $11(30 \%)$ & \\
\hline$>1,000$ & $69(55 \%)$ & $60(52 \%)$ & $14(38 \%)$ & \\
\hline Male & $69(55 \%)$ & $63(54 \%)$ & $18(49 \%)$ & 0.78 \\
\hline CRIB-II score & $9(6-11)$ & $9(6-13)$ & $10(7-13)$ & 0.105 \\
\hline PDA (treated) & $9(7.2 \%)$ & $13(11.2 \%)$ & $7(18.9 \%)$ & 0.115 \\
\hline NEC & $2(2 \%)$ & $6(5 \%)$ & $2(5 \%)$ & 0.27 \\
\hline IVH (any) & $28(22 \%)$ & $31(27 \%)$ & $16(43 \%)$ & 0.04 \\
\hline Vasopressors (yes/no) & $9(7 \%)$ & $18(15 \%)$ & $10(27 \%)$ & 0.005 \\
\hline Apgar's score (1 min) & $7(5-7)$ & $5(2-7)$ & $4(2-6)$ & $<0.001$ \\
\hline Apgar's score (5 min) & $8(6-8)$ & $7(6-8)$ & $6(5-7)$ & 0.002 \\
\hline \multicolumn{5}{|l|}{ DCC outcomes } \\
\hline Hct (birth) & $44.6(40.8-50.4)$ & $45.7(41.4-50.2)$ & $42.8(38.7-46.2)$ & 0.020 \\
\hline Hct $(48-72 \mathrm{~h})$ & $43.5(38.6-47.9)$ & $42.0(36.7-47.5)$ & $39.9(38.4-42.3)$ & 0.051 \\
\hline No. of blood transfusions $<28$ days & $0(0-2)$ & $1(0-5)$ & $1(0-4)$ & 0.01 \\
\hline \multicolumn{5}{|l|}{ Kidney outcomes } \\
\hline Initial creatinine & $0.7(0.6-0.8)$ & $0.7(0.6-0.8)$ & $0.7(0.6-0.8)$ & 0.26 \\
\hline Peak creatinine & $0.9(0.7-1.0)$ & $0.8(0.8-1.0)$ & $0.9(0.8-1.1)$ & 0.58 \\
\hline Indomethacin dose & $0(0-0.3)$ & $0(0-0.3)$ & $0(0-0.3)$ & 0.14 \\
\hline Gentamicin (no. of doses) & $2(0-4)$ & $4(2-4)$ & $2(2-6)$ & 0.029 \\
\hline
\end{tabular}

Abbreviations: c-section, caesarean section; CRIB, clinical risk index for babies; DCC, delayed cord clamping; ICC, immediate cord clamping; Hct, hematocrit; IVH, intraventricular hemorrhage; NEC, necrotizing enterocolitis; PDA, patent ductus arteriosus; sCr, serum creatinine; UCM, umbilical cord milking. Note: Statistical analysis values reported as median (interquartile range) or $n(\%)$.

Previous studies, while not including AKI or $\mathrm{sCr}$ as outcomes, have reported UOP data related to different cord management techniques. In 2009, Hosono et al compared the UOP of 40 VLBW infants and found that the UOP was higher in the UCM group for each of the first 3 days after birth compared with the ICC group. ${ }^{17}$ The $\mathrm{sCr}$ measurements were not reported, making it impossible to determine if the enrolled infants developed AKI. In 2015, a study comparing DCC to UCM, 
Table 3 AKI outcomes

\begin{tabular}{|c|c|c|c|c|c|}
\hline & $\begin{array}{l}\text { DCC } \\
n=125(\%)\end{array}$ & $\begin{array}{l}\text { ICC } \\
n=116(\%)\end{array}$ & $\begin{array}{l}\text { UCM } \\
n=37 \text { (\%) }\end{array}$ & $p$-Value (DCC-ICC) & p-Value (3 groups) \\
\hline \multicolumn{6}{|l|}{ AKI (<7 days) } \\
\hline $\mathrm{AKI}$ by $\mathrm{sCr}$ & $18(14.4 \%)$ & $16(13.8 \%)$ & $5(13.5 \%)$ & 0.892 & 0.986 \\
\hline AKI by UOP & $13(10.4 \%)$ & $15(12.9 \%)$ & $2(5.4 \%)$ & 0.540 & 0.430 \\
\hline AKI by both & $6(4.8 \%)$ & $6(5.2 \%)$ & $1(2.7 \%)$ & 0.894 & 0.822 \\
\hline AKI by either & $25(20.0 \%)$ & $25(21.6 \%)$ & $6(16.2 \%)$ & 0.767 & 0.779 \\
\hline Stage 1 & $22(17.6 \%)$ & $24(20.7 \%)$ & $4(10.8 \%)$ & \multirow[t]{3}{*}{0.553} & \multirow[t]{3}{*}{0.356} \\
\hline Stage 2 & $3(2.4 \%)$ & $1(0.9 \%)$ & $2(5.4 \%)$ & & \\
\hline Stage 3 & $0(0 \%)$ & $0(0 \%)$ & $0(0 \%)$ & & \\
\hline
\end{tabular}

Abbreviations: AKI, acute kidney injury; DCC, delayed cord clamping; ICC, immediate cord clamping; sCr, serum creatinine; UCM, umbilical cord milking; UOP, urine output.

Table 4 Regression analysis comparing DCC to ICC

\begin{tabular}{|l|l|l|l|l|}
\hline & Unadjusted & Adjusted & Adjusted by stepwise & Adjusted by BMA \\
\hline Early AKI & & & & \\
\hline AKI by sCr & $0.95(0.46-0.97)$ & $0.68(0.30-1.52)$ & $0.81(0.37-1.76)$ & $0.77(0.34-1.75)$ \\
\hline AKI by UOP & $1.28(0.58-2.82)$ & $1.11(0.48-2.52)$ & $1.32(0.57-3.04)$ & $1.26(0.56-2.83)$ \\
\hline AKI by Both & $1.08(0.34-3.45)$ & $0.48(0.19-1.21)$ & $0.97(0.28-3.43)$ & $0.96(0.28-3.29)$ \\
\hline AKI by Either & $1.10(0.59-2.05)$ & $0.86(0.44-1.69)$ & $0.89(0.45-1.75)$ & $0.93(0.46-1.86)$ \\
\hline
\end{tabular}

Abbreviations: AKI, acute kidney injury; DCC, delayed cord clamping; ICC, immediate cord clamping; sCr, serum creatinine; UOP, urine output; BMA, Bayesian model averaging.

by Katheria et al, reported that infants in the UCM group had higher UOP compared with the DCC group in the first 24 hours after birth (4.42 vs. $3.99 \mathrm{~mL} / \mathrm{kg} / \mathrm{h}, p<0.05) .{ }^{18}$ This statistically, but not clinically, significant difference does not reach the minimum rate of UOP to qualify for the KDIGO neonatal AKI criteria. We did not assess the total UOP in our study but did evaluate for clinically significant decreases in UOP over 6-hour stretches during the first week and did not find a difference between the DCC, ICC, or UCM groups.

While AKI rates were not different, based on the different cord management groups we studied, potential explanations for these findings warranted further discussion. If AKI is influenced by placental-to-neonatal transfusion volume, then AKI rates may have been unaffected because of insufficient DCC transfusion volumes, an explanation raised by the higher postdelivery hematocrits in the ICC group. However, by 48 to 72 hours the DCC group had higher hematocrits and also required less blood transfusion, findings which suggest that the DCC transfusion volumes were not the reason for lack of a difference in AKI rates. Another unaccounted factor that may have resulted in different AKI rates in our study was the use of medications other than indomethacin. First, different caffeine administration rates, previously shown to be protective of AKI in VLBW infants ${ }^{19,20}$ may have impacted AKI rates. Second, higher rates of gentamicin doses received in the first week for the ICC group may have increased the prevalence of AKI in this group disproportionately to the DCC group making it difficult to detect an effect of cord clamping. While our study did not demonstrate that DCC is associated with less AKI compared with ICC, our study supports a lack of harm associated with DCC in preterm VLBW infants. Because placental transfusion strategies have become the standard of care, the question becomes which placental transfusion strategy (DCC vs. UCM) confers improved kidney outcomes in preterm infants.

When evaluating the UCM group it is important to know that the clinical indication for infants receiving UCM was they were perceived to be "too sick" to delay the neonatal resuscitation during DCC. Therefore, the UCM cohort in our study should be viewed as the sickest group, evidenced by the lowest Apgar's scores, lower birth weights, higher use of vasopressors, higher CRIB-II scores, and lower gestational ages compared with the other groups. Despite being the highest risk group for AKI compared with the ICC and DCC groups, AKI rates were not significantly different in UCM group which suggests that UCM may have "rescued" or preserved renal blood flow, resulting in similar kidney outcomes in these infants. Future studies comparing DCC to UCM in infants who require active resuscitation after delivery, who would typically be most at risk for AKI, should consider evaluating renal function and UOP data to determine which placental transfusion strategy results in improved kidney outcomes.

\section{Limitations}

This study has several limitations, primarily due to the retrospective study design. First, AKI assessment was limited by the number of sCr's obtained by the clinician's typical practice. Second, the assessment of urine output in neonates 
is challenging and is done through weighing of diapers, potentially including stool, and thus artificially increasing the suspected urine output and allowing for "missed" cases of AKI. Third, the type of cord management was also at the clinician's discretion and, although there were small overt group differences, subtle biases may have also been present that could have affected outcomes. Fourth, we did not collect neonatal information on sepsis, but we did collect data on clinically significant hypotension requiring vasopressors and this would be the most common cause of AKI secondary to sepsis. While sepsis has been shown to influence rates of AKI, typically it is more common in smaller and lower gestational age neonates, which were equally distributed between our three cohorts of patients. Although vasopressor exposure was different in the groups, we were unable to assess the adequacy of hypotensive therapies. Lastly, the modified KDIGO definition for neonatal AKI has been challenged, particularly in the preterm group given their issues with kidney immaturity and maternal $\mathrm{sCr} .{ }^{21}$ As the neonatal AKI definition evolves, long-term and larger studies should assess whether DCC provides renal protective effects in preterm neonates.

\section{Conclusion}

In conclusion, in this retrospective study, the effect of various cord management styles was evaluated to determine the effect on kidney function in a large cohort of preterm neonates. No significant differences were found in AKI rates in the first week after birth between the DCC, UCM, and ICC groups. In our cohort, the increased hematocrit at 48 hours of age and decreased number of blood transfusions in the DCC group compared with the ICC group suggest that DCC was more effective in enhancing circulating blood volume. Overall, since the AKI incidence was not increased in the DCC or UCM groups, DCC and UCM appear safe from a neonatal kidney perspective. If further prospective, randomized trials are performed comparing DCC to UCM, kidney function data, both short (AKI and UOP) and long term (function and chronic kidney disease), should be included as a priori outcomes.

\section{Funding}

This project was completed with no specific funding. J.R.C. is funded by Thelma $\mathrm{R}$ Swotzel Collaborative Research Award, R01DK110622 and R01DK111861.

\section{Conflict of Interest}

J.R.C. is co-owner of Sindri Technologies, LLC.

\section{References}

1 Katheria AC, Lakshminrusimha S, Rabe H, McAdams R, Mercer JS. Placental transfusion: a review. J Perinatol 2017;37(02):105-111

2 Weiner GM, Zaichkin J. Textbook of Neonatal Resuscitation 7th ed. Elk Grove Village, IL: American Academy of Pediatrics and American Heart Association; 2016
3 Wyllie J, Perlman JM, Kattwinkel J, et al; Neonatal Resuscitation Chapter Collaborators. Part 7: neonatal resuscitation: 2015 international consensus on cardiopulmonary resuscitation and emergency cardiovascular care science with treatment recommendations. Resuscitation 2015;95:e169-e201

4 Committee on Obstetric Practice. Committee opinion no. 684: delayed umbilical cord clamping after birth. Obstet Gynecol 2017; 129(01):e5-e10

5 Fogarty M, Osborn DA, Askie L, et al. Delayed vs early umbilical cord clamping for preterm infants: a systematic review and metaanalysis. Am J Obstet Gynecol 2018;218(01):1-18

6 Backes $\mathrm{CH}$, Rivera BK, Haque $\mathrm{U}$, et al. Placental transfusion strategies in very preterm neonates: a systematic review and meta-analysis. Obstet Gynecol 2014;124(01):47-56

7 Nagano N, Saito M, Sugiura T, Miyahara F, Namba F, Ota E. Benefits of umbilical cord milking versus delayed cord clamping on neonatal outcomes in preterm infants: A systematic review and meta-analysis. PLoS One 2018;13(08):e0201528

8 Zappitelli M, Ambalavanan N, Askenazi DJ, et al. Developing a neonatal acute kidney injury research definition: a report from the NIDDK neonatal AKI workshop. Pediatr Res 2017;82(04):569-573

9 Jetton JG, Guillet R, Askenazi DJ, et al; Neonatal Kidney Collaborative. Assessment of Worldwide Acute Kidney Injury Epidemiology in Neonates: Design of a Retrospective Cohort Study. Front Pediatr 2016;4:68

10 Jetton JG, Boohaker LJ, Sethi SK, et al; Neonatal Kidney Collaborative (NKC). Incidence and outcomes of neonatal acute kidney injury (AWAKEN): a multicentre, multinational, observational cohort study. Lancet Child Adolesc Health 2017;1(03):184-194

11 Charlton JR, Boohaker L, Askenazi D, et al. Incidence and risk factors of early onset neonatal acute kidney injury. Clin J Am Soc Nephrol 2019;14(02):184-195

12 Daga A, Dapaah-Siakwan F, Rajbhandari S, Arevalo C, Salvador A. Diagnosis and risk factors of acute kidney injury in very low birth weight infants. Pediatr Neonatol 2017;58(03):258-263

13 Rabe H, Diaz-Rossello JL, Duley L, Dowswell T. Effect of timing of umbilical cord clamping and other strategies to influence placental transfusion at preterm birth on maternal and infant outcomes. Cochrane Database Syst Rev 2012;(08):CD003248

14 Initiative KDOQ. KDIGO clinical practice guidelines for acute kidney injury. Kidney Int Suppl 2012;2:1-138

15 Sethi SK, Raghunathan V, Shah S, et al. Fluid overload and renal angina index at admission are associated with worse outcomes in critically ill children. Front Pediatr 2018;6:118

16 Oh W, Oh MA, Lind J. Renal function and blood volume in newborn infant related to placental transfusion. Acta Paediatr 1966;55 (02):197-210

17 Hosono S, Mugishima H, Fujita H, et al. Blood pressure and urine output during the first $120 \mathrm{~h}$ of life in infants born at less than 29 weeks' gestation related to umbilical cord milking. Arch Dis Child Fetal Neonatal Ed 2009;94(05):F328-F331

18 Katheria AC, Truong G, Cousins L, Oshiro B, Finer NN. Umbilical cord milking versus delayed cord clamping in preterm infants. Pediatrics 2015;136(01):61-69

19 Carmody JB, Harer MW, Denotti AR, Swanson JR, CharltonJR. Caffeine exposure and risk of acute kidney injury in a retrospective cohort of very low birth weight neonates. J Pediatr 2016;172:63-68.e1

20 Harer MW, Askenazi DJ, Boohaker LJ, et al; Neonatal Kidney Collaborative (NKC). Association between early caffeine citrate administration and risk of acute kidney injury in preterm neonates: results from the AWAKEN study. JAMA Pediatr 2018;172(06):e180322

21 Gupta C, Massaro AN, Ray PE. A new approach to define acute kidney injury in term newborns with hypoxic ischemic encephalopathy. Pediatr Nephrol 2016;31(07):1167-1178 